\section{Investigating aggressive styles and defense mechanisms in bipolar patients and in their parents}

\section{Nicola Luigi Bragazzi, ${ }^{1}$ Franca Pezzoni, ${ }^{2}$ Giovanni Del Puente3}

1School of Public Health, Department of Health Sciences, University of Genoa;

2Department of Mental Health, Genoa

Health Local Unit;

3 Department of Neuroscience,

Rehabilitation, Ophthalmology, Genetics, Maternal and Child Health, Section of

Psychiatry, University of Genoa, Italy

\section{Abstract}

Bipolar disorder (BD) is a very common mental health disorder, whose etiology concerning aggressive styles and defense mechanisms is still poorly known despite the efforts dedicated to develop psychological and biological theories. After obtaining written signed informed consent, this study will recruit inpatients with a clinical diagnosis of $\mathrm{BD}$, based on Structured Clinical Interview and the Diagnostic and Statistical Manual of Mental Disorders criteria, and their parents. The BusPerry Aggression Questionnaire, the Defense Style Questionnaire 40, the Symptom check list SCL-90-R, developed by DeRogatis will be administered to the participants, together with a semi-structured questionnaire concerning demographic data (age, gender, employment, education) and only for the patients clinical information (onset year of the disorder, presence of co-morbidities, alcohol and drug use, suicide tendencies, kind of treatment). All the questionnaires are in the Italian validated version. The successful completion of this study will shed light on the relationship between aggressive styles and defensive mechanisms in bipolar inpatients and in their parents, helping the clinicians to develop ad hoc psychological interventions.

\section{Background}

Bipolar disorder (BD, termed also as bipolar affective disorder, or manic-depressive disorder, or manic depression) is a very common mental health disorder, characterized by a fluctuating change and swing in internal mood states and with a tremendous psychological and economic burden. 1

According to the Diagnostic and Statistical
Manual of Mental Disorders (DSM-IV-TR), BD

I has an alternation of manic and major depressive episodes, while BD II is characterized by the alternation of major depressive and hypo-manic episodes. Cyclothimia and BD nototherwise-specified (BD NOS) also belong to the bipolar spectrum.

Despite extensive investigations, BD etiology concerning aggressive styles and defense mechanisms is still poorly known: many biological theories (such as neurotransmitters impairment, alteration of physiological pathways, dysregulation of the expression of some genes, and so on) and psychological hypothesis (concerning patient's beliefs, self-esteem and self-concepts, early life events, stressors, affect regulation, and so on) have been put forward but an integrative framework is lacking, apart from few proposals. ${ }^{2}$

In this study protocol, we intend to investigate the relationship between aggressive styles and defensive mechanisms, a correlation that has been rarely explored in the literature both in BD patients and in their parents.

Since BD is a highly debilitating disorder, understanding the psychological mechanism underlying the BD pathogenesis could help clinicians to design effective psychological and psycho-therapeutic interventions, and the patients to better cope with the disease.

\section{Methods}

This protocol is currently under the examination of the International Review Board (IRB) of San Martino Hospital and all the hospitals and health local units (HLU, AUSL in Italian) taking part into the study.

The primary aim of this study is to investigate the relationship between aggressive styles and defense mechanisms both in bipolar patients and in their parents. Secondary outcomes will be: differences between BD I and BD II, correlations with clinical symptoms and SCL90-R sub-scales scores, as well as age and gender effects.

\section{Inclusion/exclusion criteria}

All the inpatients in psychiatric wards in the metropolitan area of Genoa will be invited to take part into the study. Inclusion criteria: i) to suffer from Bipolar disorder (both I and II), with a clinical diagnosis of BD based on Structured Clinical Interview (SCID) and the DSM-IV-TR criteria, ii) to be able to fill in written questionnaires and have an understanding of Italian language, iii) to have living parents.

\section{Measurements}

After obtaining written signed informed consent, the Bus-Perry Aggression Questionnaire (AGQ), the Defense Style
Correspondence: Nicola Luigi Bragazzi, School of Public Health, Department of Health Sciences (DISSAL), University of Genoa, Via Pastore 1, 16132 Genoa, Italy.

Tel.: +39.010.353.7664 - Fax: +39.010 .353 .7669$

E-mail: robertobragazzi@gmail.com

Key words: aggression questionnaire; bipolar disorder; defense style questionnaire; SCL90-R.

Contributions: the authors contributed equally.

Conflict of interests: the authors declare no potential conflict of interests.

Received for publication: 31 March 3014.

Revision received: 15 June 2014.

Accepted for publication: 15 June 2014.

This work is licensed under a Creative Commons Attribution NonCommercial 3.0 License (CC BYNC 3.0).

(C) Copyright N.L. Bragazzi et al., 2014

Licensee PAGEPress, Italy

Health Psychology Research 2014; 2:1546

doi:10.4081/hpr.2014.1546

Questionnaire 40 (DSQ-40), the Symptom check list SCL-90-R, developed by DeRogatis (SCL90-R) will be administered to the participants, together with a semi-structured questionnaire concerning demographic data (age, gender, employment, education) and (only for the BD patients) clinical information (onset year of the pathology, ICD9 code of the disorder, presence of co-morbidities, alcohol and drug use, kind of treatment, suicide tendencies). This second part should be filled in by the psychiatrist. All the questionnaires are in the Italian validated version.

\section{Bus-Perry Aggression Questionnaire}

Aggressive behaviors are frequently observed in BD patients, with a prevalence up to $25.34 \%$ in BD I. ${ }^{3}$ Aggression is a clinically relevant symptom, that can range from irritable agitation to pure destructive actions and that contributes to exacerbate the prognosis and the stigmatization of the disorder, besides being a sign of danger for the patient himself/herself and for the others.

The Buss-Perry Aggression Questionnaire, developed by Arnold Buss and Mark Perry, ${ }_{4}$ consists of 29 items which are scored using a 5 point Likert-type scale ranging from extremely uncharacteristic of me to extremely characteristic of me. The scores are normalized on a scale of 0 to 1 , with 1 being the highest level of aggression and finally four factors are identified (Physical Aggression, Verbal Aggression, Anger, and Hostility). 


\section{Defense Style Questionnaire 40}

In psychoanalysis, defense styles are considered as adaptive and protective means against stressors and unpleasant events. Generally speaking, they are regarded as being specific to neurotic disorders, even though they can be exploited and thus found also in other diseases.

Initially theorized by Sigmund and Anna Freud, Abraham and Hartmann, they have received a systematic classification by Kernberg (neurotic versus borderline/psychotic defense styles), who coined the controversial expression of splitting strategies (that is to ssay, active coping mechanisms).

Abraham was one of the first scholars to stress the importance of defensive styles in BD patients: the so-called manic-defense hypothesis. ${ }^{5}$ Abraham claimed that BD would exhibit different defensive styles from depression to an array of common difficulties, and that there are indeed some peculiar behaviors and defensive and coping strategies strictly specific of mania (such as risk-taking or other immature actions). Other scholars have related defense styles with coping strategies, ${ }^{6}$ stressors, ${ }^{7}$ patient's social cognition, ${ }^{8}$ symptoms severity, prognosis and therapeutic alliance..$^{9}$ Scholars have noticed that in BD inpatients there is a prevalence of immature defensive mechanisms, such as the creation of a false Ego and narrative. 10

In our study, we employed the brief version of DSQ, which is a self-reported inventory, which included a varied array of defensive strategies, listed according to three factors (mature, immature and neurotic styles). ${ }^{11}$ The defense mechanisms belonging to the mature style are: humor, suppression, anticipation and sublimation. The mechanisms classified as being immature are: rationalization, autistic fantasy, displacement, isolation, dissociation, splitting, devaluation, denial, somatization, passive aggression, acting out and projection. The immature strategies are: reaction formation, idealization, undoing and pseudo-altruism. The questionnaire consists of 40 items which are scored using a Likert-type scale ranging from 1 (strong disagreement) to 9 (strong agreement). DSQ-40 was chosen because, being relatively brief, was flexible, but preserving good internal consistency as measured by Cronbach's alpha coefficients (being excellent for the immature defense styles)

\section{The Symptom check list SCL-90-R}

SCL90-R, developed by Derogatis includes 90 items addressing various somatic and psychological signs of distress. ${ }^{12}$ These items are scored using a Likert-type scale from 0 (not at all) to 4 (very much). The instrument is composed of 10 sub-scales, including the general symptomatic index (GSI, score ranging from 0 to 4), which is a mean rated over all symptoms. Clinical cut-off score is $55 \%$.

\section{Statistical analysis}

Statistical analysis (namely, analysis of variance, correlation and regression) will be performed in $\mathrm{R}$ environment and using ad hoc software.

\section{Time schedule}

Preparations for recruitment of the participants, measurements will start in Summer 2013 and will be completed within 2016 .

\section{Limitations}

No control group will be recruited in this study.

\section{Conclusions}

The successful completion of this study will shed light on the relationship between aggressive styles and defensive mechanisms both in bipolar inpatients and in their parents, helping the clinicians to develop ad hoc psychological interventions.

\section{References}

1. Carta MG, Aguglia E, Balestrieri M, et al. The lifetime prevalence of bipolar disorders and the use of antidepressant drugs in bipolar depression in Italy. J Affect
Disord 2012;136:775-80.

2. Mansell W, Morrison AP, Reid G, et al. The interpretation of, and responses to, changes in internal states: an integrative cognitive model of mood swings and bipolar disorders. Behav Cogn Psychotherapy 2007;35:515-39.

3. Látalová K. Bipolar disorder and aggression. Int J Clin Pract 2009;63:889-99.

4. Buss AH, Perry MP. The aggression questionnaire. J Pers Soc Psychol 1992;63:4529.

5. Abraham K. Notes on the psycho-analytical investigation and treatment of manicdepressive insanity and allied conditions. In: Abraham K, ed. Selected papers on Psychoanalysis. New York: Basic Books; 1911. 1st edition.

6. Kramer U. Defence and coping in bipolar affective disorder: stability and change of adaptational processes. Br J Clin Psychol 2010;49:291-306.

7. Perry JC. A prospective study of life stress, defenses, psychotic symptoms, and depression in borderline and antisocial personality disorders and bipolar type ii affective disorder. J Pers Disord 1988;2:4959.

8. Lyon HM, Startup M, Bentall RP. Social cognition and themanic defense: attributions, selective attention, and self-schema in bipolar affective disorder. J Abnormal Psychol 1999;108:273-82.

9. Kramer U, de Roten Y, Perry JC, Despland JN. Specificities of defense mechanisms in bipolar affective disorder: relations with symptoms and therapeutic alliance. J Nerv Ment Dis 2009;197:675-81.

10. Baruch G. The manic defence in analysis: the creation of a false narrative. Int $\mathrm{J}$ Psychoanal 1997;78;549-59.

11. Andrews G, Singh M, Bond M. The defense style questionnaire. J Nerv Ment Dis 1993;181:246-56.

12. Derogatis LR. Symptom checklist-90revised: administration scoring and procedures manual 3rd ed. Minneapolis: MN National Computers Systems; 1994. 